Dermatomyositis (DM) is defined as an autoimmune inflammatory disease that affects the skin, the blood vessels and the muscles. It typically presents with erythema affecting mostly the eyes and the hands as well as proximal muscle weakness. It has been also correlated with various types of cancer, including ovarian cancer. A systematic PubMed and Scopus search was conducted. A total of 110 women were included in our review. The median age of the patients was 52.5 years (8-85). The most frequent histological type of malignancy was epithelial (87 cases, $79.1 \%$ ) and in only one patient $(0.9 \%)$ DM was co-existing with a cancer recurrence. A clinical diagnosis of DM or PM preceded the diagnosis of ovarian cancer in $69.1 \%$ (76/110), while paraneoplastic DM after the diagnosis of ovarian cancer was reported in 31\% (34/110). Serum antibodies were present in $22.5 \%$ $(25 / 110)$ and the median creatine kinase during first evaluation was 886 (56.6-16,596). Postoperative improvement of the symptoms was observed in $24.6 \%(27 / 110)$ while also $24.6 \%$ (27/110) needed post-treatment rheumatological management. Neoadjuvant chemotherapy or radiotherapy was necessary in 58.2\% (64/110) and a cancer recurrence was identified in $28.2 \%$ (31/110), with a median follow up of 24.5 months (5-210) Finally, 52 (47.3\%) deaths were reported in a median follow-up period of 16 months (0-210). It is crucial that DM patients should receive a thorough evaluation for ovarian cancer, among other malignancies, encompassing an abdominal CT or MRI scan and serum Ca-125 marker measurements. Treatment of ovarian cancer is usually accompanied by remission of DM symptoms in most of the cases.

Key words: dermatomyositis, polymyositis, ovarian cancer, paraneoplastic syndrome.

Contemp Oncol (Pozn) 2020; 24 (4): 252-257 DOI: https://doi.org/10.5114/wo.2020.102814

\section{Dermatomyositis and/or polymyositis as a paraneoplastic manifestation of ovarian cancer: a systematic review}

\author{
Victoria Psomiadou', Ioannis D. Gkegkes ${ }^{2}$, Christos lavazzo ${ }^{1}$
}

${ }^{1}$ Gynaecological Oncology Department, Metaxa Cancer Hospital, Piraeus, Greece ${ }^{2}$ Department of Colorectal Surgery, Royal Devon and Exeter NHS Foundation Trust, Exeter, United Kingdom

\section{Introduction}

Dermatomyositis (DM) is an uncommon idiopathic inflammatory myositis presenting principally with heliotrope rush, Gottron's papules and various dermatological manifestations including erythema and telangiectasia among others [1]. Polymyositis (PM) is an idiopathic inflammatory myopathy as well, characterized by symmetric muscle weakness and accompanied by a high skeletal muscle enzyme serum level and diagnosed through histopathological and electromyographical findings [2]. Myositis in general was related to malignancy as early as in 1916 by Sterz et al., who described it as a paraneoplastic syndrome. Paraneoplastic manifestations include a wide variety of diseases, mainly categorized in four large groups, consisting of endocrinological, hematological, neurological and dermatological symptoms, which fail to be attributed to effects of the primary tumor itself, its metastases or hormones produced by the tissues affected [3]. Although the type of malignancy varies with PM complicating mainly Hodgkin's lymphoma while DM is usually associated with ovarian. breast, colorectal, lung, breast, pancreas, stomach and prostate. The mechanism behind this relationship seems to lie on the vicious circle of activation of the complement system resulting in endomuscular capillaries lysis and further inflammation due to muscle ischemia [4]. The incidence of DM is rather low affecting $0.5-0.89$ per 100,000 patients, female predominating male with a ratio $2: 1$ [5]. DM and PM respectively are highly associated with the risk of cancer development, which may precede or coincide with the diagnosis or follow it in cases of paraneoplastic DM/PM. Breast, lung, colorectal and nasopharyngeal cancer have been among others mostly correlated with the presence of inflammatory myositis while PM specifically is related to Hodgkin lymphoma and other types of lymphoma [6]. Ovarian cancer and DM have been described to coexist [1]. Based on our findings, 110 similar cases have been reported in the literature. In a study by Cheng et al., the risk of developing myositis is low but its peak lies in an interval of 3 years before or after the diagnosis of ovarian cancer, since the disease can precede, coexist or follow the malignancy [1].

In this study, we conducted a systematic review of the literature aiming to present the relationship between ovarian cancer and DM or PM, with the ultimate purpose to set ovarian cancer among the underlying causes of idiopathic inflammatory myopathies and therefore suggest a diagnostic workup for ovarian malignancy in DM or PM patients. 


\section{Material and methods}

\section{Data sources}

We conducted a systematic search in PubMed and Scopus up to May 2020 using the key words: (ovarian cancer) and (dermatomyositis or polymyositis or myositis) as search terms. The search was performed by two independent authors VP and IDG. The inclusion criteria were well specified and no discrepancies in search results were found.

\section{Study selection criteria}

We reviewed all studies presenting a relationship between ovarian cancer and DM and PM. The inclusion criteria were: patients with ovarian cancer diagnosis that presented with DM and/or PM. The search string is described in Fig. 1. Articles published before 1980, studies written in languages other than English, German and Greek, book chapters, review articles, letters/editorials/conference papers as well as surveys and animal studies were not included in our study. Letters that did not mention any correlation between and ovarian cancer to DM or vice versa, have been excluded from our analysis. Furthermore, 45 articles were excluded after detailed screening according to specific criteria (Fig. 1). Preferred Reporting Items for Systematic Reviews and Meta-Analyses (PRISMA) guidelines were followed and carrying out at this systematic review [7].

\section{Definitions}

Clinical improvement was defined as an immediate postoperative improvement during the hospitalization of the patient.

\section{Results}

\section{Selected studies}

We retrieved a total of 135 studies. 45 articles were considered to be eligible for inclusion in our review, while 90 were excluded.

Table 1 summarizes the data collected from studies presenting cases with ovarian cancer coexisting with an inflammatory myopathy. Specifically, 48 scientific papers and 110 patients were studied [8-54]. The median age of the patients was 52.5 years (8-85). Regarding the oncological features of ovarian cancer, epithelial ovarian cancer (HG adenocarcinoma / serous / papillary / clear cell / endometrioid) was the major histological type of cancer in $79.1 \%$ of the cases (87/110). More specifically, serous carcinoma was diagnosed in $43.6 \%$ (48/110), papillary in $6.4 \%$ (7/110), while endometrioid in 3.6\% (4/110) and clear cell carcinoma in $21.8 \%(2 / 110)$. Interestingly, the histology in 2 patients (1.8\%) proved dysgerminoma and $3(2.7 \%)$ women suffered from a poorly differentiated malignancy. Rarely, metastatic cancer was also found in 0.9\% (1/110) and $1.8 \%(2 / 110)$ were diagnosed with mature teratoma, $0.9 \%(1 / 110)$ with transitional carcinoma, $0.9 \%$ (1/110) with signet ring carcinoma, $0.9 \%$ (1/110) with large cells carcinoma and $0.9 \%(1 / 110)$ with a malignancy of Mullerian origin. The FIGO stage of the disease at the time of the diagnosis was I in 2.7\% (3/110), II in 4.6\% (5/110), III in 59.1\% (65/110) and IV in $18.2 \%$ (20/110) patients respectively.
DM and PMf were the first manifestation of the disease in $63.6 \%(70 / 110)$ and $5.5 \%(6 / 110)$ respectively, while in $32.7 \%$ (36/110), ovarian cancer was already diagnosed and myositis was identified as a paraneoplastic syndrome. In $5.5 \%(6 / 110)$ other rheumatological (clinical amyopathic dermatomyositis (CADM), panniculitis, ichthyosis, poikiloderma, cutaneous Herpes zoster) conditions were also identified.

Regarding laboratory findings, median creatine kinase level was $886(56.6-16,596)$ and serum antibodies were present in 22.5\% (25/110) (ANA, anti-Jo1, anti-ds-DNA, anti-TIF1c, RNP, anti SS-A, anti-cardiolipin IgM, striational (Striated Muscle) antibody, P/Q-type calcium channel antibody, anti-p155/140, IgA and IgG).

The majority of the patients $(70.9 \%, 78 / 110)$ were treated with primary surgery, while $4.6 \%(5 / 110)$ underwent interval debulking surgery. Twelve women $(10.9 \%$ 12/110) were treated with chemotherapy, while in $0.9 \%(1 / 110)$ combined chemotherapy and radiotherapy was used. Three patients $(2.7 \%, 3 / 110)$ did not undertake any treatment, one because of a deadly trauma. For $10 \%$ (11/110) of the patients, data about the treatment methods were not available.

In 24.6\% (27/110) women postoperative recession of the symptoms was immediately observed, while $24.6 \%$ $(27 / 110)$ patients too as well still needed rheumatological treatment after surgery. Specifically, prednisone was used in patients $8.9 \%$ (9/110), prednisolone in 4.6\% (5/110), hydrocortisone in $0.9 \%(1 / 110)$ while steroid use in general is reported in patients $5.5 \%(6 / 110)$. Methotrexate was used in cases $4.6 \%$ (5/110) while azathioprine and intravenous immunoglobulin were used in cases 3.6\% (4/110) respectively. Other medical factors reported include tacrolimus, fluocinonide and hydroxychloroquine, all used once $(0.9 \%)$ in the so far published literature.

Neoadjuvant chemotherapy or radiotherapy was necessary in 58.2\% (64/110) and a cancer recurrence was

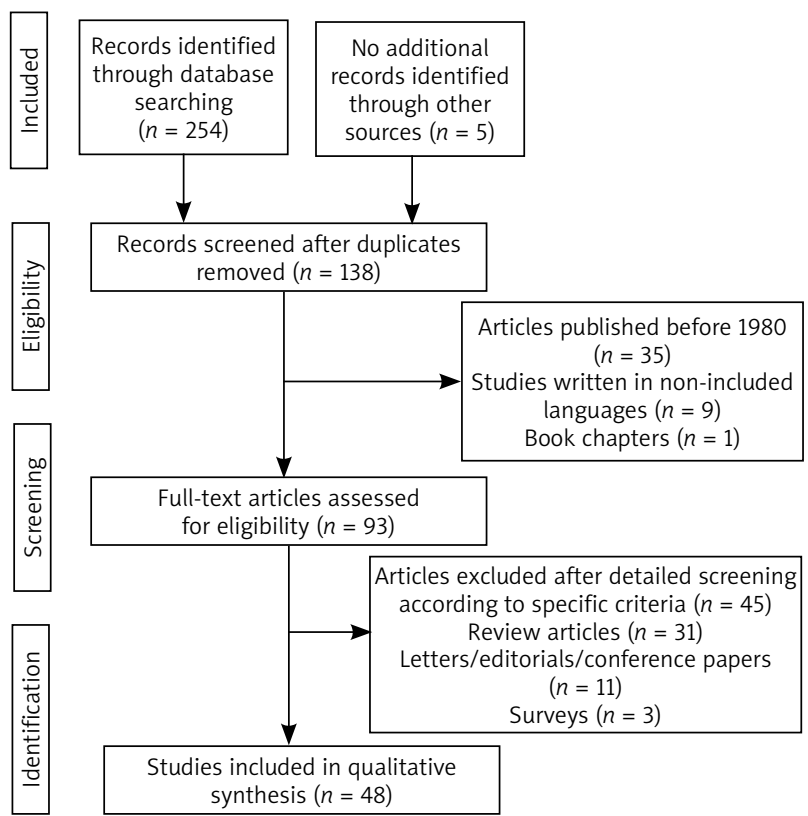

Fig. 1. Flow diagram of the selection process of articles included in the review 
Table 1. Main characteristics and outcomes of the patients with dermatomyositis and ovarian cancer

\begin{tabular}{|c|c|}
\hline Demographics & $n / N(\%)$ \\
\hline Age in years, median (range) & $52.5(8-85)$ \\
\hline \multicolumn{2}{|l|}{ Clinical features of colorectal cancer } \\
\hline \multicolumn{2}{|l|}{ Histology } \\
\hline Serous adenocarcinoma & $48 / 110(43.6)$ \\
\hline Papillary adenocarcinoma & $7 / 110(6.4)$ \\
\hline Endometrioid adenocarcinoma & 4/110 (3.6) \\
\hline Clear cell adenocarcinoma & 2/110 (1.8) \\
\hline Dysgerminoma & $2 / 110(1.8)$ \\
\hline Mature teratoma & $2 / 110(1.8)$ \\
\hline Poorly differentiated & $3 / 110(2.7)$ \\
\hline Transitional carcinoma & $1 / 110(0.9)$ \\
\hline Signet ring cells carcinoma & $1 / 110(0.9)$ \\
\hline Large cells carcinoma & $1 / 110(0.9)$ \\
\hline Malignancy of Mullerian origin & $1 / 110(0.9)$ \\
\hline \multicolumn{2}{|l|}{ FIGO stage } \\
\hline I & $3 / 110(2.7)$ \\
\hline$\|$ & $5 / 110(4.6)$ \\
\hline III & $65 / 110(59.1)$ \\
\hline IV & 20/110 (18.2) \\
\hline \multicolumn{2}{|l|}{ Rheumatological manifestations } \\
\hline Dermatomyositis as first manifestation & $70 / 110(63.6)$ \\
\hline Polymyositis as first manifestation & $6 / 110(5.4)$ \\
\hline Simultaneous or after OC diagnosis & $34 / 110(31)$ \\
\hline $\begin{array}{l}\text { Presence of other rheumatological } \\
\text { conditions }\end{array}$ & $6 / 110(5.5)$ \\
\hline $\begin{array}{l}\text { Creatine kinase (in } \mathrm{U} / \mathrm{L} \text { ) (at first } \\
\text { presentation), median (range) }\end{array}$ & $886(56.6-16,596)$ \\
\hline Presence of serum antibodies & $25 / 110(22.7)$ \\
\hline \multicolumn{2}{|l|}{ Outcomes } \\
\hline $\begin{array}{l}\text { Immediate improvement of symptoms } \\
\text { after surgery }\end{array}$ & $27 / 110(24.6)$ \\
\hline $\begin{array}{l}\text { Need of post-treatment rheumatological } \\
\text { treatment }\end{array}$ & $27 / 110(24.6)$ \\
\hline \multicolumn{2}{|l|}{ Type of treatment } \\
\hline Primary surgery & 78/110 (70.9) \\
\hline Interval debulking surgery & $5 / 110(4.6)$ \\
\hline Chemotherapy & $12 / 110(10.9)$ \\
\hline $\begin{array}{l}\text { Combination of chemotherapy and } \\
\text { radiotherapy }\end{array}$ & $1 / 110(0.9)$ \\
\hline Not specified & 11/110 (10) \\
\hline \multicolumn{2}{|l|}{ Postoperative oncologic treatment } \\
\hline Chemotherapy & $64 / 110(58.2)$ \\
\hline Recurrence of cancer & $31 / 110(28.2)$ \\
\hline Follow-up (in months), median (range) & $16(0-210)$ \\
\hline Death & $52(47.3)$ \\
\hline
\end{tabular}

identified in $28.2 \%$ (31/110), with a median follow up of 24.5 months (5-210). Finally, 52 (47.3\%) deaths were reported in a median follow-up period of 16 months (0-210).

\section{Discussion}

A systematic review was conducted in order to determine the association between DM and PM and ovarian cancer. According to our results, the diseases often coexist, and a full cancer screening should be considered for diagnosing ovarian cancer among patients with myositis. $D M$ is very uncommon; so, a systematic review is a useful tool to evaluate the clinical and diagnostic characteristics of the disease as well as the epidemiological features of its association with ovarian cancer. Although the number of articles included in our study is not large, the number of patients that we examined is significant, compared to the epidemiologic data of DM and concomitant ovarian cancer in the literature [55].

Furthermore, it has been found that the prognosis in DM patients with malignancy is worse since typically the stage of the disease at the time of the diagnosis is already advanced [56].

DM presents with a wide range of symptoms including painless proximal symmetrical weakness as well as weakness of the pharyngeal muscle, often leading to aspiration pneumonia and dysphagia, conditions with the worst prognosis. Other clinical features encompass gastrointestinal symptoms and more rarely involvement of the ventilatory muscles. Nevertheless, pathognomonic of the disease are the skin lesions that are observed, that be Gottron papules and heliotrope rash [57]. Gkegkes et al. in their study about colorectal cancer and paraneoplastic DM demonstrate that the symptoms usually resolve after the successful treatment of the underlying malignancy [3].

Regarding the pathogenesis of the disease, many hypotheses have been suggested, mainly of autoimmune nature against regenerating myoblasts. The inflammatory reactions in the skin and muscles, are also escorted by inflammatory autoantigens expression mediated by neoplastic and muscle cells [3]. Especially, a high level of anti-p155/140 auto-antibody, should raise suspicion for undiagnosed ovarian cancer. Microangiopathy is also observed, with muscle capillary involvement, due to presence of auto-antibodies too. After all, the diagnosis is confirmed with muscle or skin biopsies. On the one hand, skin changes include vascular dermis-epidermis lesions, inflammatory lymphocytic infiltration and perivascular infiltration while in the muscled capillary obliteration and thrombosis combined with destruction of the endothelial cells as well as the perivascular and perifasicular tissues [17].

In an analysis by Cherin et al. total ovarian cancer incidence in DM patients was $13.3 \%$, while in women aged over 40 years old, the incidence was as high as $21.4 \%$ [54]. lavazzo et al. report of a case of PM presented following the successful treatment of a recurrent double primary breast and ovarian carcinoma [2]. After all, in a study by Field et al. it is strongly recommended that all patients with presenting with DM and breast or ovarian cancers should undergo genetic testing, since a plethora of such patients 
were found to carry BRCA mutations [44]. In the present study, in 76 women, the myositis was manifested first in comparison to 36 ovarian cancer patients, who were later diagnosed with paraneoplastic DM or PM. The diagnosis was based on the clinical features and the elevated serum creatinine kinase, which had a median level of $886 \mathrm{U} / \mathrm{l}$ as well as the presence of auto-antibodies, which were found positive in 25 women. Creatine kinase (CK) is a typical laboratory marker to measure the involvement of the muscles in DM. Interestingly, in the propensity analysis by Cheng et al. [1], a correlation was found between CK level and Ca125 tumor marker level in almost 50\% of the patients with concurrence of ovarian cancer and DM that were studied. Auto-antibody anti-Jo-1 is also frequently present, among other auto-antibodies such as anti-Mi-2 and antinuclear antibodies, the last one being though non-diagnostic for the disease [58].

Regarding histology, the most common histological type of the tumors was epithelial $(79,1 \%)$ while rare cases of mature teratomas and dysgerminomas were also published [34, 37, 45, 48]. In fact, Roselino et al. [34] report a case of an 8-years-old child suffering from DM accompanied by ichthyosis which later was proven to be an underlying dysgerminoma, while a similar case of juvenile dysgerminoma and coexisting DM in a 16-year-old is described by Solomon and Maurer [37]. Except for the histology of the tumors included in our review, with an epidemiological distribution typical of the worldwide distribution of the disease, what was also in harmony with the data we found in the literature, was the advanced stage of malignancy, that most of our patients were diagnosed with. Specifically, Cheng et al. [1] in their analysis of 23 women with ovarian cancer and DM have found that $82.6 \%$ and $17.4 \%$ of them were diagnosed with FIGO stage III and IV respectively. In our study, stage II and IV were represented from 65 (59.1\%) and 20 (18.2\%) of the patients respectively, while stage I was diagnosed only in $3(2.7 \%)$ and stage II only in 5 (4.6\%) of the women included.

Despite the advanced stage, the majority of the patients 78 (70.9\%) were managed by surgical treatment, followed by chemotherapy or radiotherapy in 64 of them (58.2\%), while the rest of the patients were basically treated with chemotherapy, alone or with consequent interval debulking surgery. Whichever treatment, resolution of DM was observed at the same rate as in the cases where post-treatment, rheumatological factors, typically steroids, were needed (24.6\% respectively). Tembe et al. in a study of 10 patients with paraneoplastic DM and PM, reports 3 cases with advanced stage ovarian adenocarcinoma, all treated with chemotherapy, who demanded post-treatment therapy with a combination of methotrexate, azathioprine and steroids [51].

The course of the patients studied varied in terms of survival and recurrence rated. In total, patients were followed for a period of 24.5 months and death is reported in 52 cases (47.3\%). These findings are in agreement with those of a propensity score analysis by Chen et al., where overall survival as well as 5 -year progression-free rates of patients with ovarian cancer and DM were poorer in comparison with ovarian cancer patients not suffering from
DM (71.6\% vs. $51.8 \%, p=0.02$ and $30.5 \%$ vs. $0 \%, p=0.018$ accordingly) [1]. However, an interesting case is reported by Girouard et al., where a 63-years old patient with metastatic ovarian cancer and DM-associated panniculitis, was diagnosed with 4 recurrences and was still alive after a 210-months follow up, the longest in the present study [18]. On the other hand, Davis et al. and Aydi et al. both report cases of immediate death after the cancer diagnoses $[13,35]$, the last one in a patient with rare histology of a signet ring cell carcinoma. In total, patients were followed for a period of 24.5 months and death is reported in 52 cases $(47.3 \%)$.

Our results indicate that although rare, the prevalence of underlying ovarian cancer in patients with DM/PM is significant, so a thorough screening for an occult malignancy should be conducted, including in female patients an abdominal CT or MRI scan and serum levels of Ca-125 tumor marker, independent of the age of the patient. Recent clinical guidelines indicate additionally to Ca-125 levels, a pelvic examination and a transvaginal ultrasound every $6-12$ months for $2-5$ years after the diagnosis of DM is set [5]. Nevertheless, larger studies should be done in order to form universal guidelines with regards to the cancer screening in DM/PM patients.

To our knowledge, this is the first systematic review in the literature regarding the association of DM and PM and ovarian neoplasia. A detailed search of the published studies by two independent authors aimed in the elimination of the data loss. However, a number of limitations should be taken under consideration, before drawing conclusions. First of all, the number of the patients included is small, as a result of the limited number of relevant studies published. Furthermore, many studies were written in languages other than English and thus excluded from our review, while the included studies were all retrospective and individual presentation of random cases was prevalent. Another limitation is the fact that the diagnosis of DM is both rare and difficult, thus, restricting the cases presented in the literature in total. As a result, further, larger, well-designed studies are necessary to determine the optimal cancer screening for female patients with DM and/or PM.

\section{Conclusions}

DM and/or PM commonly relate to ovarian cancer, although the pathogenesis of both the disease itself and its association with ovarian malignancy is not completely understood yet. Hence, it is crucial that a full cancer screening including abdominal depictions and Ca-125 level measurements should be conducted in any DM/PM female patient, with a high suspicion for ovarian neoplasia. Moreover, in women with diagnosed ovarian carcinoma and emerging symptoms of DM/PM, a thorough check for a cancer recurrence is needed. Regarding the treatment of DM, cancer treatment should be attempted first, since in the majority of the cases, the symptoms resolve consequently.

The authors declare no conflict of interest. 


\section{References}

1. Cheng H, Huo L, Wang D, Xiang Y. Concurrence of ovarian cancer and dermatomyositis: a propensity score analysis. J Gynecol Oncol 2019; 30: e99.

2. Iavazzo C, Vorgias G, Papadakis M, Manikis P, Mavromatis I, Akrivos T. Polymyositis in a patient with recurring ovarian cancer and history of unrelated breast cancer. Arch Gynecol Obstet 2007; 276: 81-84.

3. Hudson CN, Curling M, Potsides P, Lowe DG. Paraneoplastic syndromes in patients with ovarian neoplasia. J R Soc Med 1993; 86: 202-204.

4. Gkegkes ID, Minis EE, lavazzo C. Dermatomyositis and colorectal cancer: a systematic review. Ir J Med Sci 2018; 187: 615-620.

5. Field C, Goff BA. Dermatomyositis - key to diagnosing ovarian cancer, monitoring treatment and detecting recurrent disease: Case report. Gynecol Oncol Rep 2017; 23: 1-3.

6. Jakubaszek M, Kwiatkowska B, Maślińska M. Polymyositis and dermatomyositis as a risk of developing cancer. Reumatologia 2015; 53: 101-105.

7. Moher D, Liberati A, Tetzlaff J, et al. Preferred reporting items for systematic reviews and meta-analyses: the PRISMA statement. PLoS Med 2009; 6: e1000097.

8. Vílchez-Márquez F, Prieto-Llamas R, Sánchez-Sánchez G. Dermatomyositis associated with ovarian cancer. Med Clin (Barc) 2017; 148: e55.

9. Ikeda S, Arita M, Misaki K, et al. Incidence and impact of interstitial lung disease and malignancy in patients with polymyositis, dermatomyositis, and clinically amyopathic dermatomyositis: a retrospective cohort study. Springerplus 2015; 4: 240.

10. Seidler AM, Wasserman DI, González-Serva A, Konnikov N. Amyopathic dermatomyositis resembling stasis dermatitis. J Am Acad Dermatol 2008; 59: 515-518.

11. el-Azhary RA, Pakzad SY. Amyopathic dermatomyositis: retrospective review of 37 cases. J Am Acad Dermatol 2002; 46: 560-565.

12. Flynn M, Ottaway Z, Kaur J, Waters J. Three differently timed presentations of dermatomyositis associated with advanced ovarian cancer. BMJ Case Rep 2018; 2018: pii: bcr-2017-222627.

13. Davis MD, Ahmed I. Ovarian malignancy in patients with dermatomyositis and polymyositis: a retrospective analysis of fourteen cases. J Am Acad Dermatol 1997; 37: 730-733.

14. Arshad I, Barton D. Dermatomyositis as a paraneoplastic phenomenon in ovarian cancer. BMJ Case Rep 2016; 2016: pii: bcr2016215463.

15. Callen JP. Dermatomyositis and female malignancy. J Surg Oncol 1986; 32: 121-124.

16. Whitmore SE, Rosenshein NB, Provost TT. Ovarian cancer in patients with dermatomyositis. Medicine (Baltimore) 1994; 73: 153 160.

17. Christie A, McKay N, Nussey F. Dermatomyositis as presenting feature of ovarian cancer, treated with neo-adjuvant chemotherapy and interval debulking surgery. Gynecol Oncol Case Rep 2013; 6: 13-15.

18. Girouard SD, Velez NF, Penson RT, Massarotti EM, Vleugels RA. Panniculitis associated with dermatomyositis and recurrent ovarian cancer. Arch Dermatol 2012; 148: 740-744.

19. Ben-Zvi N, Shani A, Ben-Baruch G, et al. Dermatomyositis following the diagnosis of ovarian cancer. Int J Gynecol Cancer 2005; 15: 1124-1126.

20. Teraishi M, Nakajima K, Ishimoto T, et al. Anti-transcription intermediary factor $1 \gamma$ antibody titer correlates with clinical symptoms in a patient with recurrent dermatomyositis associated with ovarian cancer. Int J Rheum Dis 2018; 21: 900-902.

21. Voravud N, Dimopoulos M, Hortobagyi G, Ross M, Theriault R. Breast cancer a second primary ovarian cancer in dermatomyositis. Gynecol Oncol 1991; 43: 286-290.

22. Requena C, Alfaro A, Traves V, et al. Paraneoplastic dermatomyositis: a study of 12 cases. Actas Dermosifiliogr 2014; 105: 675-682

23. Hong MK, Lee MH, Ding DC, Chu SC, Chu TY. High grade serous ovarian carcinoma with serous tubal intraepithelial carcinoma in a case presented with atypical glandular cell favor neoplasm cervi- cal cytology and dermatomyositis. Taiwan J Obstet Gynecol 2015; 54: 183-186

24. Raffel GD, Gravallese EM, Schwab P, Joseph JT, Cannistra SA. Diagnostic dilemmas in oncology: case 2. Dermatomyositis and ovarian cancer. J Clin Oncol 2001; 19: 4341-4343.

25. Chao LW, Wei LH. Dermatomyositis as the initial presentation of ovarian cancer. Taiwan J Obstet Gynecol 2009; 48: 178-180.

26. Venhuizen AC, Martens JE, van der Linden PJ. Dermatomyositis as first presentation of ovarian cancer. Acta ObstetGynecol Scand 2006; 85: 1271-1272.

27. Valverde R, Sánchez-Caminero MP, Calzado L, et al. [Dermatomyositis and punctate porokeratotic keratoderma as paraneoplastic syndrome of ovarian carcinoma]. Actas Dermosifiliogr 2007; 98: 358-360.

28. Kubo M, Sato S, Kitahara H, Tsuchida T, Tamaki K. Vesicle formation in dermatomyositis associated with gynecologic malignancies. J Am Acad Dermatol 1996; 34: 391-394.

29. Nakanishi K, Cualing H, Husseinzadeh N. Dermatomyositis as a presenting symptom of ovarian cancer. Obstet Gynecol 1999; 94: 836-838.

30. Lin C, Ying Z, Sijing C. Spontaneous resolution of dermatomyositis associated with fallopian-tube carcinoma following staging surgery: A case report. Medicine (Baltimore) 2019; 98: e14530.

31. Hagman JH, Bianchi L, Campione E, Vidolin AP, Chimenti S. Dermatomyositis associated with ovarian transitional cell carcinoma. J Am Acad Dermatol 2001; 45: 642-644.

32. Verducci MA, Malkasian GD Jr, Friedman SJ, Winkelmann RK. Gynecologic carcinoma associated with dermatomyositis-polymyositis. Obstet Gynecol 1984; 64: 695-698.

33. Ghosh A, Malak TM, Pool AJ. Polymyositis and ovarian carcinoma: a case report. Arch Gynecol Obstet 2007; 275: 195-197.

34. Roselino AM, Souza CS, Andrade JM, et al. Dermatomyositis and acquired ichthyosis as paraneoplastic manifestations of ovarian tumor. Int J Dermatol 1997; 36: 611-614.

35. Aydin AA, Ayik H, Onder AH, Ozturk B, Yildiz M. A diagnostic dilemma: A rare case of an ovarian carcinoma with signet ring cells hidden behind a substantial dermatomyositis. J Cancer Res Ther 2019; 15: 1395-1397.

36. Kalogiannidis I, Papanikolaou A, Xanthakis I, Makedos A, Prapas N. Clear cell ovarian carcinoma following polymyositis diagnosis: a case report and review of the literature. Hippokratia 2008; 12: 181-185.

37. Solomon SD, Maurer KH. Association of dermatomyositis and dysgerminoma in a 16-year-old patient. Arthritis Rheum 1983; 26 : 572-573.

38. Mahajan C, Ray C, Singhal A, et al. Dermatomyositis as a Paraneoplastic Manifestation of Ovarian Cancer: A Case Report and Review of Literature. Indian J Gynecol Oncolog 2020; 18: 12.

39. Peters WA 3rd, Andersen WA, Thornton WN Jr. Dermatomyositis and coexistent ovarian cancer: a review of the compounding clinical problems. Gynecol Oncol 1983; 15: 440-446.

40. Maoz CR, Langevitz P, Livneh A, et al. High incidence of malig nancies in patients with dermatomyositis and polymyositis: an 11-year analysis. Semin Arthritis Rheum 1998; 27: 319-324.

41. Cai Y, Qiao J, Fang H. Metachronous multiple primary malignant neoplasms in a patient with dermatomyositis. Indian J Dermatol Venereol Leprol 2012; 78: 665.

42. Dieffenbach C, Bouberhan S, Raynor E, Shea M, Liu F. Polymyositis as a presentation of advanced carcinoma of Mullerian origin: A case report and discussion. Gynecol Oncol Rep 2018; 25: 1-2.

43. Cadena I, Werth VP, Levine P, et al. Lasting pathologic complete response to chemotherapy for ovarian cancer after receiving antimalarials for dermatomyositis. Ecancermedicalscience 2018; 12: 837.

44. Field C, Goff BA. Dermatomyositis - key to diagnosing ovarian can cer, monitoring treatment and detecting recurrent disease: Case report. Gynecol Oncol Rep 2017; 23: 1-3.

45. Yu X, Qin D, Ma D, Yao Q. Adult dermatomyositis associated with benign ovarian teratoma: A case report. Oncol Lett 2016; 11: 2611 2614.

46. Amer S, Qureshi W, Hassan S. Rash: An initial presentation of ovarian cancer. Indian J Cancer 2015; 52: 713-714. 
47. Eliaz I. The Role of Galectin-3 as a Marker of Cancer and Inflammation in a Stage IV Ovarian Cancer Patient with Underlying Pro-Inflammatory Comorbidities. Case Rep Oncol 2013; 6: 343-349.

48. Chen, G., Lee, S. Two fatal cases of dermatomyositis and ovarian cancer. Hong Kong J Dermatol Venereol 2011; 19: 80-85.

49. Ibarra M, Chou P, Pachman LM. Ovarian teratoma mimicking features of juvenile dermatomyositis in a child. Pediatrics 2011; 128 : e1293-e1296.

50. Choudry A, Pamma G, Ali M. Ovarian cancer and dermatomyositis. Internet J Oncol 2009; 7: 1.

51. Tembe AG, Ramteke S, Joshi VR, Balakrishnan C. Dermatomyositis/ polymyositis associated with malignancy: our experience with ten patients and review of relevant literature. Int J Rheum Dis 2008; 11: 269-273.

52. Parker, J, Sykes P, Rome R. Dermatomyositis and Ovarian Cancer: A Rare Occurrence but a Significant Association. Aust N Z J Obstet Gynaecol 1994; 34: 616-617.

53. Mordel N, Margalioth EJ, Harats N, Ben-Baruch N, Schenker JG. Concurrence of ovarian cancer and dermatomyositis. A report of two cases and literature review. J Reprod Med 1988; 33: 649-655.

54. Cherin P, Piette JC, Herson S, et al. Dermatomyositis and ovarian cancer: a report of 7 cases and literature review. J Rheumatol 1993; 20: 1897-1899.

55. Trallero-Araguás E, Rodrigo-Pendás JÁ, Selva-O'Callaghan A, et al. Usefulness of anti-p155 autoantibody for diagnosing cancer-associated dermatomyositis: a systematic review and meta-analysis. Arthritis Rheum 2012; 64: 523-532.

56. Liu Y, Xu L, Wu H, et al. Characteristics and predictors of malig nancy in dermatomyositis: Analysis of 239 patients from northern China. Oncol Lett 2018; 16: 5960-5968.

57. Thompson C, Piguet V, Choy E. The pathogenesis of dermatomyositis. Br J Dermatol 2018; 179: 1256-1262.

58. Strowd LC, Jorizzo JL. Review of dermatomyositis: establishing the diagnosis and treatment algorithm. J Dermatolog Treat 2013; 24: 418-42

\section{Address for correspondence}

\section{loannis D. Gkegkes}

Department of Colorectal Surgery

Royal Devon and Exeter NHS Foundation Trust Exeter EX2 5DW, United Kingdom

e-mail: ioannisgkegkes@gmail.com

Submitted: 25.08 .2020

Accepted: 22.10 .2020 